\title{
POINCARÉ FAMILIES OF $G$-BUNDLES ON A CURVE
}

\author{
INDRANIL BISWAS AND NORBERT HOFFMANN
}

\begin{abstract}
Let $G$ be a reductive group over an algebraically closed field $k$. Consider the moduli space of stable principal $G$-bundles on a smooth projective curve $C$ over $k$. We give necessary and sufficient conditions for the existence of Poincaré bundles over open subsets of this moduli space, and compute the orders of the corresponding obstruction classes. This generalizes the previous results of Newstead, Ramanan and Balaji-Biswas-Nagaraj-Newstead to all reductive groups, to all topological types of bundles, and also to all characteristics.
\end{abstract}

\section{INTRODUCTION}

Let $C$ be a compact Riemann surface of genus $g \geq 2$. Let $\mathfrak{M}_{n, d}$ denote the coarse moduli space of stable vector bundles $E$ over $C$ of fixed rank $n \geq 2$ and degree $d \in \mathbb{Z}$. Newstead proved that there is no Poincaré bundle over $C \times \mathfrak{M}_{n, 0}$, more precisely, the universal projective bundle over $C \times \mathfrak{M}_{n, 0}$ cannot be lifted to a topological vector bundle 21. Ramanan proved that if $n$ and $d$ have a common divisor, then there is no Poincaré bundle over $C \times U$ for any Zariski-open subset $\emptyset \neq U \subseteq \mathfrak{M}_{n, d}$ [22]. If $n$ is coprime to $d$, it is easy to see that there is a Poincaré bundle over $C \times \mathfrak{M}_{n, d}$.

Now let $G$ be a complex semisimple group with one simple factor. Let $\mathfrak{M}_{G}^{0, \text { rs }}$ be the moduli space of topologically trivial regularly stable principal $G$-bundles over $C$ (a stable $G$-bundle is called regularly stable if its automorphism group coincides with the center of $G$ ). The main theorem of 1 s says that there is a Poincaré bundle (also called universal principal $G$-bundle) over $C \times \mathfrak{M}_{G}^{0, r s}$ if and only if the center of $G$ is trivial. The proof in 1 is a generalization of the proof in [21.

In this paper, we address the existence of such Poincaré bundles for all topological types, not just topologically trivial. The definition of Poincaré bundle (or Poincaré family) is recalled in Definition 6.1. We give necessary and sufficient conditions for their existence over the whole regularly stable locus (Corollary 6.7), as well as over arbitrarily small Zariski-open subsets of it (Corollary 6.9). In fact we compute the orders of the corresponding obstruction classes, which live in appropriate Brauer groups; cf. Theorem 6.6 and Theorem 6.8. These orders are given in terms of the root system of $G$ (see Section 6 ). They are easy to compute for any given reductive group $G$. The results are valid also in positive characteristics.

The proof of our results is based on the observation that the regularly stable locus in the moduli stack of principal $G$-bundles is a gerbe over the corresponding locus in the coarse moduli scheme. The Brauer class of this gerbe is precisely the obstruction

2000 Mathematics Subject Classification. 14D22.

The first author thanks the Freie Universität Berlin for hospitality. The second author was supported by the SFB 647: Raum - Zeit - Materie. 
class mentioned above. We have studied the Picard groups of such moduli stacks in [3. This is used in Section 7 to determine the order of the obstruction class.

In order to use this method, we need to know that the regularly stable locus is at least non-empty. This is already known in characteristic zero, but apparently not in positive characteristic. We recall the notion of regular stability in Section 2, and formulate the precise statement that we need as Theorem 2.5. Its proof is given in Section 5 , using some preparations in Sections 3 and 4 .

\section{Regularly stable $G$-Bundles}

Let $C$ be a connected smooth projective algebraic curve of genus $g \geq 2$ over an algebraically closed base field $k$. Throughout this text, we assume that $G$ is a smooth connected linear algebraic group over $k$. The Lie algebra of $G$ will be denoted by $\mathfrak{g}$.

Let $\mathcal{M}_{G}$ denote the moduli stack of principal $G$-bundles on $C$. One knows that $\mathcal{M}_{G}$ is an Artin stack, locally of finite type and smooth over $k$. Its decomposition into connected components

$$
\mathcal{M}_{G}=\coprod_{d \in \pi_{1}(G)} \mathcal{M}_{G}^{d}
$$

is indexed by the fundamental group $\pi_{1}(G)$ of $G$; see [17, Section 5]. Let $G_{u} \subseteq G$ be the unipotent radical. Choosing a maximal torus $T$ in the reductive quotient $G / G_{u}$, the group $\pi_{1}(G)$ is by definition the quotient of $\operatorname{Hom}\left(\mathbb{G}_{\mathrm{m}}, T\right)$ modulo its subgroup generated by the coroots of $G / G_{u}$.

Assume that $G$ is reductive. According to [18, Proposition 3.20], the stable and the semistable principal $G$-bundles form open substacks

$$
\mathcal{M}_{G}^{d, \mathrm{~s}} \subseteq \mathcal{M}_{G}^{d, \mathrm{ss}} \subseteq \mathcal{M}_{G}^{d} \text {. }
$$

Lemma 2.1. The complement of $\mathcal{M}_{G}^{d, \mathrm{~s}}$ has codimension $\geq g-1$ in $\mathcal{M}_{G}^{d}$.

Proof. Let $\mathcal{C}$ be an irreducible component of the complement $\mathcal{M}_{G}^{d} \backslash \mathcal{M}_{G}^{d, \mathrm{~s}}$. Let $K$ be an algebraically closed field containing $k$, and let $E$ be a principal $G$-bundle on $C \otimes_{k} K$ whose classifying morphism $\operatorname{Spec}(K) \rightarrow \mathcal{M}_{G}$ maps onto the generic point of $\mathcal{C}$. Then $E$ is not stable, so there is a parabolic subgroup $P \subseteq G$ and a reduction of $E$ to a principal $P$-bundle $F$ with $\operatorname{deg}(\operatorname{ad}(F)) \leq 0$; here $\operatorname{ad}(F):=F \times{ }^{P} \mathfrak{p}$ denotes the vector bundle obtained from $F$ via the adjoint representation ad $: P \rightarrow \operatorname{Aut}(\mathfrak{p})$, where $\mathfrak{p}$ is the Lie algebra of $P$. We consider the connected component

$$
\mathcal{M}_{P}^{\delta} \subseteq \mathcal{M}_{P}
$$

that contains $F$. Extending the structure group defines a representable 1-morphism $\mathcal{M}_{P}^{\delta} \rightarrow \mathcal{M}_{G}^{d}$ which is dominant onto $\mathcal{C}$. Hence

$$
\operatorname{dim} \mathcal{C} \leq \operatorname{dim} \mathcal{M}_{P}^{\delta}=(g-1) \operatorname{dim} P-\operatorname{deg}(\operatorname{ad}(F)) \leq(g-1) \operatorname{dim} P .
$$

So the codimension of $\mathcal{C}$ in $\mathcal{M}_{G}^{d}$ is $\geq(g-1)(\operatorname{dim} G-\operatorname{dim} P) \geq g-1$.

Due to [12, 13] and [14, 15], there is a projective coarse moduli scheme

$$
\pi: \mathcal{M}_{G}^{d, \mathrm{ss}} \longrightarrow \mathfrak{M}_{G}^{d}
$$

of semistable principal $G$-bundles $E$ on $C$ of type $d \in \pi_{1}(G)$. These constructions also show that the stable locus $\mathcal{M}_{G}^{d, \mathrm{~s}} \subseteq \mathcal{M}_{G}^{d, \mathrm{ss}}$ is the inverse image of an open subscheme $\mathfrak{M}_{G}^{d, \mathrm{~s}} \subseteq \mathfrak{M}_{G}^{d}$. 
Let $Z \subseteq G$ denote the (scheme-theoretic) center. It is a group scheme of the form $Z \cong \mathbb{G}_{\mathrm{m}}^{r} \times \mu_{n_{1}} \times \cdots \times \mu_{n_{s}}$ with $r, s \geq 0$ and $n_{1}, \ldots, n_{s} \geq 1$.

Definition 2.2. A stable principal $G$-bundle $E$ on $C$ is regularly stable if the canonical morphism

$$
Z \longrightarrow \operatorname{Aut}(E)
$$

into the group scheme of global automorphisms of $E$ is an isomorphism.

Proposition 2.3. The locus of regularly stable principal G-bundles

$$
\mathcal{M}_{G}^{d, \mathrm{rs}} \subseteq \mathcal{M}_{G}^{d, \mathrm{~s}}
$$

is an open substack. It is the inverse image of an open subscheme

$$
\mathfrak{M}_{G}^{d, \mathrm{rs}} \subseteq \mathfrak{M}_{G}^{d, \mathrm{~s}} .
$$

In order to prove this proposition, we recall Luna's étale slice theorem [20, and its generalization to positive characteristic in 2 .

Let $H$ be a smooth reductive algebraic group over $k$. Suppose that $H$ acts on an affine variety $X$ of finite type over $k$. Let $X / H$ denote the GIT-quotient. Let $x \in X(k)$ be a closed point. Its orbit $H \cdot x$ is a smooth subvariety of $X$. The orbit map $H \rightarrow H \cdot x$ is an fppf-locally trivial principal bundle under the scheme-theoretic stabilizer $H_{x} \subseteq H$.

An $H_{x}$-stable affine subvariety $S \subseteq X$ with $x \in S$ is called an étale slice at $x$ if the commutative diagram of canonical maps

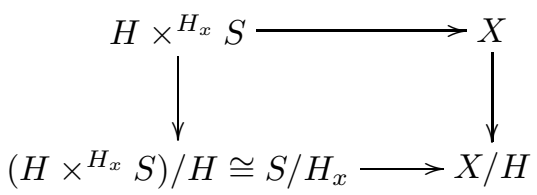

is cartesian, and the horizontal maps are both étale.

Proposition 2.4. If the orbit $H \cdot x$ is closed in $X$ and the stabilizer $H_{x}$ is linearly reductive, then there exists an étale slice at $x$.

Proof. Bardsley and Richardson have proved this under the assumption that the orbit map $H \rightarrow H \cdot x$ is separable [2, Proposition 7.6]. However, this assumption is not needed in their argument if one works with the (possibly non-reduced) schemetheoretic stabilizer $H_{x}$.

More precisely, [2, Proposition 7.5] allows us to assume without loss of generality that $X$ is a vector space over $k$, and that $H$ acts linearly on $X$. Since $H_{x}$ is linearly reductive, there is an $H_{x}$-stable affine linear subspace $S \subseteq X$ containing $x$ such that $S$ and the orbit $H \cdot x$ are transversal at $x$. This means that the canonical morphism of smooth varieties

$$
H \times{ }^{H_{x}} S \longrightarrow X
$$

is étale at the point $(1, x) \in H \times{ }^{H_{x}} S$. Using [2, Theorem 6.2], this implies that some Zariski-open neighborhood of $x$ in $S$ is an étale slice at $x$.

Proof of Proposition [2.3. According to the construction in [12, 13, the stable locus $\mathfrak{M}_{G}^{d, \mathrm{~s}}$ is a GIT-quotient of a quasiprojective scheme $X$ over $k$ modulo a reductive group $H$. More precisely, the quotient morphism

$$
X \longrightarrow \mathfrak{M}_{G}^{d, \mathbf{s}}
$$


is affine, its fibers are precisely the $H$-orbits, the $H$-invariant open subschemes of $X$ are precisely the inverse images of open subschemes in $\mathfrak{M}_{G}^{d, \mathrm{~s}}$, and the stack quotient $[X / H]$ is the moduli stack $\mathcal{M}_{G}^{d, \mathrm{~s}}$.

In particular, every orbit $H \cdot x \subseteq X$ with $x \in X(k)$ is closed, and the schemetheoretic stabilizer $H_{x}$ always contains $Z$. If $H_{x}$ is just $Z$, then it is in particular linearly reductive [8, II, $\S 2,2.5]$, so Proposition 2.4 applies and shows that there is an étale slice at $x$.

It follows that the set of all points $x \in X(k)$ with stabilizer $H_{x}=Z$ is Zariskiopen. Since it is also $H$-invariant, it defines an open substack $\mathcal{M}_{G}^{d, \mathrm{rs}} \subseteq \mathcal{M}_{G}^{d, \mathrm{~s}}$ and an open subscheme $\mathfrak{M}_{G}^{d, \mathrm{rs}} \subseteq \mathfrak{M}_{G}^{d, \mathrm{~s}}$.

Theorem 2.5. We still assume that $G$ is reductive.

i) If $g \geq 3$, then $\mathcal{M}_{G}^{d, \mathrm{rs}}$ is non-empty.

ii) If $g \geq 4$, then the complement of $\mathcal{M}_{G}^{d, \mathrm{rs}}$ in $\mathcal{M}_{G}^{d}$ has codimension at least two.

In characteristic 0 , this is proved in [10, Theorem II.6], with better bounds on $g$. However, we were not able to generalize the cited proof to positive characteristic, mainly due to difficulties with finite unipotent automorphism groups. After some preparations in Sections 3 and 4 , we prove Theorem 2.5 in Section 5 .

\section{BRILL-NOETHER LOCI}

By a $G$-module $V$, we mean a finite-dimensional vector space $V$ over $k$ together with a morphism $G \rightarrow \mathrm{GL}(V)$ of algebraic groups over $k$. Given such a $G$-module $V$, we can associate to each principal $G$-bundle $E$ on $C$ the vector bundle $E \times{ }^{G} V$ on $C$ with fiber $V$. The Brill-Noether locus

$$
\mathcal{W}_{V}:=\left\{[E]: \mathrm{H}^{0}\left(C, E \times{ }^{G} V\right) \neq 0\right\} \subseteq \mathcal{M}_{G}
$$

is closed, according to the semicontinuity theorem. This section deals with codimension estimates for such loci.

Each group homomorphism $\varphi: H \rightarrow G$ induces, by extension of the structure group, a 1-morphism $\varphi_{*}: \mathcal{M}_{H} \rightarrow \mathcal{M}_{G}$. Moreover, $\varphi$ allows us to turn $G$-modules $V$ into $H$-modules. We note that $\mathcal{W}_{V} \subseteq \mathcal{M}_{H}$ is the inverse image of $\mathcal{W}_{V} \subseteq \mathcal{M}_{G}$ in this situation.

For a character $\chi: G \rightarrow \mathbb{G}_{\mathrm{m}}$, let $\ell_{\chi}$ be the resulting 1-dimensional $G$-module. Let $\langle\chi, d\rangle \in \mathbb{Z}$ denote the image of $d \in \pi_{1}(G)$ under the group homomorphism $\chi_{*}: \pi_{1}(G) \rightarrow \pi_{1}\left(\mathbb{G}_{\mathrm{m}}\right)=\mathbb{Z}$.

Lemma 3.1. If $\chi \neq 0$, then $\mathcal{W}_{\ell_{\chi}}$ has codimension $\geq g-\langle\chi, d\rangle$ in $\mathcal{M}_{G}^{d}$.

Proof. We start with the special case $G=\mathbb{G}_{\mathrm{m}}$ and $\chi=\mathrm{id}$. Note that $\mathcal{W}_{\ell_{\text {id }}} \subseteq \mathcal{M}_{\mathbb{G}_{\mathrm{m}}}^{d}$ is empty for $d<0$, and for $d \geq 0$ it corresponds to the image of the Abel-Jacobi map $C^{d} \rightarrow \operatorname{Pic}^{d}(C)$. This image has dimension $\leq d$, and hence codimension $\geq g-d$ in $\operatorname{Pic}^{d}(C)$. Thus $\mathcal{W}_{\ell_{\mathrm{id}}}$ has codimension $\geq g-d$ in $\mathcal{M}_{\mathbb{G}_{\mathrm{m}}}^{d}$, as claimed.

In the general case, $\mathcal{W}_{\ell_{\chi}} \subseteq \mathcal{M}_{G}$ is the inverse image of $\mathcal{W}_{\ell_{\mathrm{id}}} \subseteq \mathcal{M}_{\mathbb{G}_{\mathrm{m}}}$ under the induced 1-morphism $\chi_{*}: \mathcal{M}_{G} \rightarrow \mathcal{M}_{\mathbb{G}_{\mathrm{m}}}$. Using the above special case, it thus suffices to show that this 1-morphism $\chi_{*}$ is flat.

If a closed normal subgroup $N \subseteq G$ is smooth, then the canonical 1-morphism $\mathcal{M}_{G} \rightarrow \mathcal{M}_{G / N}$ is also smooth by deformation theory. We apply this to the reduced identity component $N:=\operatorname{ker}(\chi)^{0} \subseteq G$. It contains the unipotent radical $G_{u} \subseteq G$ 
and the commutator subgroup $[G, G] \subseteq G$, so $G / N$ is a torus. The character $G / N \rightarrow \mathbb{G}_{\mathrm{m}}$ induced by $\chi$ is nontrivial and has finite kernel, so it is an isogeny. In particular, $G / N$ has rank 1, and the induced 1-morphism $\mathcal{M}_{G / N} \rightarrow \mathcal{M}_{\mathbb{G}_{\mathrm{m}}}$ is flat. It follows that the composition $\chi_{*}: \mathcal{M}_{G} \rightarrow \mathcal{M}_{G / N} \rightarrow \mathcal{M}_{\mathbb{G}_{m}}$ is also flat, as required.

For the rest of this section, we consider the group $G:=\mathbb{G}_{a} \rtimes \mathbb{G}_{\mathrm{m}}$ for the standard action of $\mathbb{G}_{\mathrm{m}}$ on $\mathbb{G}_{a}$. Let $\pi: G \rightarrow \mathbb{G}_{\mathrm{m}}$ denote the projection.

Put $q=1$, or let $q$ be a power of $p$ if $\operatorname{char}(k)=p \geq 2$. Let $\wp^{q}$ denote the 2-dimensional $G$-module given by the homomorphism $G \rightarrow \mathrm{GL}_{2}$ that sends $t \in \mathbb{G}_{a}$ to $\left(\begin{array}{ll}1 & 0 \\ t^{q} & 1\end{array}\right)$, and $h \in \mathbb{G}_{\mathrm{m}}$ to $\left(\begin{array}{ll}1 & 0 \\ 0 & h^{q}\end{array}\right)$. We have an exact sequence

$$
0 \longrightarrow \ell_{q \pi} \longrightarrow \wp^{q} \longrightarrow \ell_{0} \longrightarrow 0
$$

of $G$-modules. In the case $q=1$, we write $\wp:=\wp^{1}$; here we get in particular an exact sequence of $G$-modules

$$
0 \longrightarrow \ell_{\pi} \longrightarrow \wp \longrightarrow \ell_{0} \longrightarrow 0 .
$$

It yields an equivalence of categories between principal $G$-bundles on $C$ and exact sequences of vector bundles on $C$

$$
0 \longrightarrow L \longrightarrow E \longrightarrow \mathcal{O}_{C} \longrightarrow 0
$$

with $\operatorname{rank}(L)=1$ and $\operatorname{rank}(E)=2$. Thus the fiber of $\pi_{*}: \mathcal{M}_{G} \rightarrow \mathcal{M}_{\mathbb{G}_{\mathrm{m}}}$ over a line bundle $L$ on $C$ parameterizes extensions of $\mathcal{O}_{C}$ by $L$.

The type $d \in \pi_{1}(G)=\mathbb{Z}$ of a principal $G$-bundle on $C$ is the degree of the corresponding line bundle $L$.

Lemma 3.2. The locus $\mathcal{W}_{\wp} \backslash \mathcal{W}_{\ell_{\pi}} \subseteq \mathcal{M}_{G}$ has dimension $g-1$.

Proof. Let a line bundle $L$ on $C$ with $\mathrm{H}^{0}(C, L)=0$ be given, and an extension $E$ of $\mathcal{O}_{C}$ by $L$ as above. Then $\mathrm{H}^{0}(C, E) \neq 0$ if and only if this extension splits. Hence the restriction of the 1-morphism $\pi_{*}$ is an isomorphism of $\mathcal{W}_{\wp} \backslash \mathcal{W}_{\ell_{\pi}}$ onto $\mathcal{M}_{\mathbb{G}_{\mathrm{m}}} \backslash \mathcal{W}_{\ell_{\mathrm{id}}}$.

Corollary 3.3. If $d \leq 0$, then $\mathcal{W}_{\wp}$ has codimension $\geq g-1$ in $\mathcal{M}_{G}^{d}$.

Proof. Since its fibers parameterize extensions by $\mathcal{O}_{C}$, the 1-morphism $\pi_{*}: \mathcal{M}_{G}^{d} \rightarrow$ $\mathcal{M}_{\mathbb{G}_{\mathrm{m}}}^{d}$ is smooth of relative dimension $g-1-d$; see Lemma 2.10 of [16. Hence $\mathcal{M}_{G}^{d}$ has dimension $2 g-2-d$.

If $d \leq 0$, then $\mathcal{W}_{\wp} \backslash \mathcal{W}_{\ell_{\pi}}$ has codimension $\geq g-1$ in $\mathcal{M}_{G}^{d}$ by Lemma 3.2, and $\mathcal{W}_{\ell_{\pi}}$ has codimension $\geq g$ in $\mathcal{M}_{G}^{d}$ by Lemma 3.1

By a prime divisor $D$ in an integral Artin stack $\mathcal{M}$, we mean an integral closed substack $D \subseteq \mathcal{M}$ of codimension 1 .

Lemma 3.4. Suppose $\operatorname{char}(k)=p \geq 2$, and $d \leq 0$.

i) If $g \geq 2$, then the complement of $\mathcal{W}_{\wp^{p}}$ in $\mathcal{M}_{G}^{d}$ is non-empty.

ii) If $g \geq 3$, and some prime divisor $D \subseteq \mathcal{M}_{G}^{d}$ is contained in $\mathcal{W}_{\wp}$, then $D=\left(\pi_{*}\right)^{-1}\left(D^{\prime}\right)$ for some prime divisor $D^{\prime} \subseteq \mathcal{M}_{\mathbb{G}_{\mathrm{m}}}^{d}$.

iii) If $p=2$, then $\mathcal{W}_{\wp^{2}}$ has codimension $\geq g-1$ in $\mathcal{M}_{G}^{d}$. 
Proof. We consider a principal $G$-bundle on $C$ whose moduli point is in $\mathcal{W}_{\wp^{p}}$, but not in $\mathcal{W}_{\wp} \cup \mathcal{W}_{\ell_{p \pi}}$. The exact sequences $0 \rightarrow \ell_{\pi} \rightarrow \wp \rightarrow \ell_{0} \rightarrow 0$ and $0 \rightarrow \ell_{p \pi} \rightarrow$ $\wp^{p} \rightarrow \ell_{0} \rightarrow 0$ of $G$-modules induce exact sequences

$$
0 \rightarrow L \longrightarrow E \longrightarrow \mathcal{O}_{C} \rightarrow 0 \quad \text { and } \quad 0 \rightarrow L^{\otimes p} \longrightarrow \text { Frob }^{*}(E) \longrightarrow \mathcal{O}_{C} \rightarrow 0
$$

of associated vector bundles on $C$, where Frob : $C \rightarrow C$ is the absolute Frobenius. Our assumption means that the extension $E$ doesn't split, but the extension Frob $^{*}(E)$ does split.

In particular, there is a nonzero morphism $\operatorname{Frob}^{*}(E) \rightarrow L^{\otimes p}$ of vector bundles on $C$. Using adjunction, we obtain a nonzero morphism

$$
\varphi: E \longrightarrow \operatorname{Frob}_{*}\left(L^{\otimes p}\right)=\operatorname{Frob}_{*}\left(\operatorname{Frob}^{*}(L)\right)=L \otimes \operatorname{Frob}_{*}\left(\mathcal{O}_{C}\right) .
$$

Since $\mathrm{H}^{0}\left(C, \operatorname{Frob}_{*}\left(\mathcal{O}_{C}\right)\right)=\mathrm{H}^{0}\left(C, \mathcal{O}_{C}\right)=k$, the restriction of $\varphi$ maps $L \subseteq E$ to the subbundle $L \subseteq L \otimes \operatorname{Frob}_{*}\left(\mathcal{O}_{C}\right)$. Thus we obtain a nonzero morphism of short exact sequences over $C$

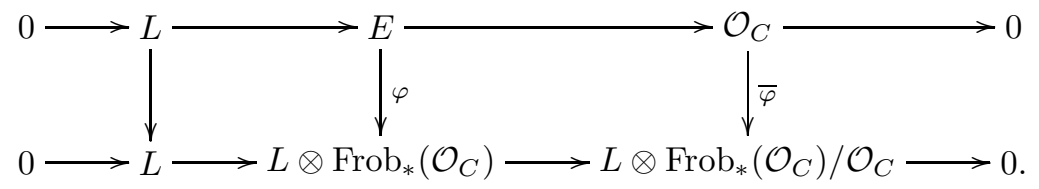

The top row doesn`t split, so $\bar{\varphi} \neq 0$. This shows

$$
\mathcal{W}_{\wp p} \subseteq \mathcal{W}_{\wp} \cup \mathcal{W}_{\ell_{p \pi}} \cup\left(\pi_{*}\right)^{-1}\left(\mathcal{W}^{\prime}\right)
$$

as closed loci in $\mathcal{M}_{G}$, where we put

$$
\mathcal{W}^{\prime}:=\left\{[L]: \mathrm{H}^{0}\left(C, L \otimes \operatorname{Frob}_{*}\left(\mathcal{O}_{C}\right) / \mathcal{O}_{C}\right) \neq 0\right\} \subseteq \mathcal{M}_{\mathbb{G}_{\mathrm{m}}} .
$$

As $d \leq 0$ by assumption, $\mathcal{W}_{\wp} \cup \mathcal{W}_{\ell_{p \pi}}$ has codimension $\geq g-1$ in $\mathcal{M}_{G}^{d}$ due to Corollary 3.3 and Lemma 3.1. Due to [23, Théorème 4.1.1], we have $\mathcal{M}_{\mathbb{G}_{\mathrm{m}}}^{0} \nsubseteq \mathcal{W}^{\prime}$ and hence $\mathcal{M}_{\mathbb{G}_{\mathrm{m}}}^{d} \nsubseteq \mathcal{W}^{\prime}$. This implies part (i) of the lemma.

In the situation of part (ii), we conclude $D \subseteq\left(\pi_{*}\right)^{-1}\left(D^{\prime}\right)$ for some irreducible component $D^{\prime}$ of $\mathcal{W}^{\prime} \cap \mathcal{M}_{\mathbb{G}_{\mathrm{m}}}^{d}$. But $D$ is a prime divisor, $\pi_{*}$ is smooth, and $D^{\prime} \neq$ $\mathcal{M}_{\mathbb{G}_{\mathrm{m}}}^{d}$. It follows that $D^{\prime}$ is also a prime divisor, and that $D=\left(\pi_{*}\right)^{-1}\left(D^{\prime}\right)$. This proves part (ii) of the lemma.

Now suppose $p=2$. Then $\operatorname{Frob}_{*}\left(\mathcal{O}_{C}\right) / \mathcal{O}_{C}$ is a line bundle on $C$, of degree $g-1$ 23. p. 118]. If $\operatorname{deg}(L)=d$, then $\bar{\varphi}$ is a nonzero section of a line bundle of degree $g-1+d$. Sending our $G$-bundle to the divisor of this section defines a morphism

$$
\mathcal{M}_{G}^{d} \cap \mathcal{W}_{\wp} \backslash\left(\mathcal{W}_{\wp} \cup \mathcal{W}_{\ell_{2 \pi}}\right) \longrightarrow C^{(g-1+d)} .
$$

This is an open embedding, since the top row in (11) can be reconstructed from $L$ and $\bar{\varphi}$ by pulling back the bottom row. Hence we conclude

$$
\operatorname{dim}\left(\mathcal{M}_{G}^{d} \cap \mathcal{W}_{\wp^{2}} \backslash\left(\mathcal{W}_{\wp} \cup \mathcal{W}_{\ell_{2 \pi}}\right)\right) \leq g-1+d .
$$

As we are assuming $d \leq 0$, part (iii) of the lemma follows. 


\section{B-MODUleS}

In this section, we assume that our algebraic group $G$ over $k$ is of adjoint type and simple. Choose a Borel subgroup $B \subseteq G$, and a maximal torus $T \subseteq B$. Let us denote by

$$
\operatorname{Hom}\left(T, \mathbb{G}_{\mathrm{m}}\right) \supset \Phi \supset \Phi^{+} \ni \alpha_{1}, \ldots, \alpha_{l}
$$

the root system of $G$ with respect to $T$, the positive roots with respect to $B$, and the simple roots, respectively. We adopt the following:

Convention 4.1. In the case $\Phi \cong G_{2}$, we number the two simple roots $\alpha_{1}, \alpha_{2} \in \Phi^{+}$ in such a way that $\alpha_{1}$ is short and $\alpha_{2}$ is long.

Every $B$-module $V$ decomposes into $T$-eigenspaces

$$
V=\bigoplus_{\chi \in \operatorname{Hom}\left(T, \mathbb{G}_{m}\right)} V_{\chi} .
$$

E. g. the adjoint action of $B \subseteq G$ on $\mathfrak{g}$ yields the Cartan decomposition

$$
\mathfrak{g}=\mathfrak{g}_{0} \oplus \bigoplus_{\alpha \in \Phi} \mathfrak{g}_{\alpha}
$$

We choose basis vectors $e_{\alpha} \in \mathfrak{g}_{\alpha}$ that form a Chevalley system; see for example [7, p. 56f.]. Given a root $\alpha \in \Phi$, [9, Exposé XXII, Théorème 1.1] provides a unique $T$-equivariant morphism of algebraic groups

$$
\exp _{\alpha}: \mathfrak{g}_{\alpha} \longrightarrow G
$$

whose derivative at 0 is the inclusion $\mathfrak{g}_{\alpha} \hookrightarrow \mathfrak{g}$. The map $\exp _{\alpha}$ is an isomorphism onto a closed subgroup, which we denote by $U_{\alpha} \subseteq G$.

Put $u_{\alpha}:=\exp _{\alpha}\left(e_{\alpha}\right) \in U_{\alpha}$. Let $U$ denote the unipotent radical of $B$; it is generated by the $U_{\alpha}$ with $\alpha \in \Phi^{+}$. For every $B$-module $V$, the action of $U_{\alpha} \subseteq B$ on $T$-eigenvectors $v \in V_{\chi}$ satisfies

$$
\exp _{\alpha}\left(t e_{\alpha}\right) \cdot v=v+\sum_{n \geq 1} t^{n} \rho_{\chi, n \alpha}(v)
$$

for all $t \in k$, with linear maps $\rho_{\chi, n \alpha}: V_{\chi} \rightarrow V_{\chi+n \alpha}$; see e.g. Lemma 5.2 of [4]. Hence the space of invariants $V^{B} \subseteq V$ is the kernel of the linear map

$$
\oplus \rho_{0, n \alpha}: V_{0} \longrightarrow \bigoplus_{\alpha \in \Phi^{+}} \bigoplus_{n \geq 1} V_{n \alpha} .
$$

Therefore, given a short exact sequence $0 \rightarrow V^{\prime} \rightarrow V \rightarrow V^{\prime \prime} \rightarrow 0$ of $B$-modules, the snake lemma yields an exact sequence of vector spaces

$$
0 \rightarrow\left(V^{\prime}\right)^{B} \longrightarrow V^{B} \longrightarrow\left(V^{\prime \prime}\right)^{B} \longrightarrow \operatorname{coker}\left(V_{0}^{\prime} \stackrel{\oplus \rho_{0, n \alpha}}{\longrightarrow} \bigoplus_{\alpha \in \Phi^{+}} \bigoplus_{n \geq 1} V_{n \alpha}^{\prime}\right)
$$

For each simple root $\alpha_{i}$, we define a homomorphism of algebraic groups

$$
\pi_{i}: B=U \rtimes T \longrightarrow \mathbb{G}_{a} \rtimes \mathbb{G}_{\mathrm{m}}
$$

as the product of $\alpha_{i}: T \rightarrow \mathbb{G}_{\mathrm{m}}$ with the projection $U \rightarrow \mathbb{G}_{a}$ that vanishes on all $U_{\alpha}$ with $\alpha \in \Phi^{+} \backslash\left\{\alpha_{i}\right\}$, and maps $\exp \left(t e_{\alpha_{i}}\right) \in U_{\alpha_{i}}$ to $t \in \mathbb{G}_{a}$.

As $G$ is of adjoint type, the product map $\prod_{i} \alpha_{i}: T \rightarrow \mathbb{G}_{\mathrm{m}}^{l}$ is an isomorphism. Thus we get an exact sequence

$$
1 \longrightarrow U^{\prime} \longrightarrow B \stackrel{\prod_{i} \pi_{i}}{\longrightarrow}\left(\mathbb{G}_{a} \rtimes \mathbb{G}_{\mathrm{m}}\right)^{l} \longrightarrow 1
$$


of algebraic groups over $k$, where $U^{\prime}$ is generated by all $U_{\alpha}$ with $\alpha \in \Phi^{+}$not simple. In particular, $U^{\prime}$ is smooth and connected.

Put $q=1$, or let $q$ be a power of $p$ if $\operatorname{char}(k)=p \geq 2$. Let $\wp_{i}^{q}$ denote the $B$-module obtained via $\pi_{i}$ from the $\left(\mathbb{G}_{a} \rtimes \mathbb{G}_{\mathrm{m}}\right)$-module $\wp^{q}$, and put $\wp_{i}:=\wp_{i}^{1}$. These are indecomposable $B$-modules of dimension 2 .

Proposition 4.2. Let $V$ be an indecomposable B-module of dimension 2. Then $V \cong \ell_{\chi} \otimes \wp_{i}^{q}$ for some character $\chi: B \rightarrow \mathbb{G}_{\mathrm{m}}$, some simple root $\alpha_{i}$, and some number $q$ as above.

Proof. Because $V$ is indecomposable of dimension 2, there is a positive root $\alpha$ such that $U_{\alpha} \subseteq B$ acts nontrivially on $V$.

Using relation (2), this observation implies $V \cong \ell_{\chi} \oplus \ell_{\chi+q \alpha}$ as $T$-modules, for some character $\chi: T \rightarrow \mathbb{G}_{\mathrm{m}}$ and some integer $q \geq 1$.

Choosing eigenvectors $v_{\psi} \in V_{\psi}$, we moreover see that $\exp \left(t e_{\alpha}\right) \in U_{\alpha}$ maps $v_{\chi}$ to $v_{\chi}+c t^{q} v_{\chi+q \alpha}$ for a constant $c \in k$. Here $c \neq 0$, as $V$ is indecomposable. Replacing $v_{\chi}$ by $c v_{\chi}$ yields $c=1$ without loss of generality.

Since the map $U_{\alpha} \rightarrow \operatorname{GL}(V)$ at hand is a group homomorphism, we have $q=1$, or $q$ is a power of $\operatorname{char}(k) \geq 2$. We claim that $\alpha$ is a simple root $\alpha_{i}$; then $V \cong \ell_{\chi} \otimes \wp_{i}^{q}$ will follow.

Suppose that $\alpha$ is not simple. Then $\alpha=\beta+\gamma$ for some $\beta, \gamma \in \Phi^{+}$. We assume without loss of generality that $\gamma$ is not longer than $\beta$. Let $n \geq 1$ be maximal such that $\beta_{0}:=\alpha-n \gamma \in \Phi^{+}$; then $n \leq 3$.

If $\gamma$ is long, then $\beta$ is also long by assumption, so $n=1$, and $\beta_{0}=\beta$ is long as well. Thus $\gamma$ is not longer than $\beta_{0}$ in any case.

Due to [9, Exposé XXIII, Proposition 6.4], the equation

$$
u_{\beta_{0}} u_{\gamma}=u_{\gamma} u_{\beta_{0}}\left(u_{\beta_{0}+\gamma}\right)^{ \pm 1}\left(u_{\beta_{0}+2 \gamma}\right)^{ \pm 1}\left(u_{\beta_{0}+3 \gamma}\right)^{ \pm 1}\left(u_{2 \beta_{0}+3 \gamma}\right)^{ \pm 1}
$$

holds in $B$, using the convention $u_{\psi}:=1$ for $\psi \notin \Phi$. One of these factors is $\left(u_{\alpha}\right)^{ \pm 1}$; it acts nontrivially on $V$ by assumption. But all other factors in this equation act trivially on $V$, due to relation (2).

This contradiction proves the claim.

Corollary 4.3. If $V$ is a $B$-module with $V^{B}=0$, then $V$ is a successive extension of $B$-modules of the form $\ell_{\chi}$ with $\chi \neq 0$, or of the form $\wp_{i}^{q}$.

Proof. Suppose $V^{B}=0$ and $V \neq 0$. We argue by induction on $\operatorname{dim} V$. As $B$ is solvable, there is a 1-dimensional $B$-submodule $V^{1} \subseteq V$. From the exact sequence (3), we see that there are two possible cases:

- $\left(V / V^{1}\right)^{B}=0$, and $V^{1} \cong \ell_{\chi}$ for some nontrivial character $\chi: T \rightarrow \mathbb{G}_{\mathrm{m}}$.

- $\operatorname{dim}\left(V / V^{1}\right)^{B}=1$, and $V^{1} \cong \ell_{n \alpha}$ for some $\alpha \in \Phi^{+}$and some $n \geq 1$.

In the second case, let $V^{2} \subseteq V$ be the inverse image of $\left(V / V^{1}\right)^{B} \subseteq V / V^{1}$. Then $\left(V^{2}\right)^{B}=0$, and $V^{2} \cong \ell_{0} \oplus \ell_{n \alpha}$ as $T$-modules. Due to Proposition 4.2, this implies $V^{2} \cong \wp_{i}^{q}$ for some $i$ and $q$. The short exact sequence

$$
0 \longrightarrow\left(V / V^{1}\right)^{B} \longrightarrow V / V^{1} \longrightarrow V / V^{2} \longrightarrow 0
$$

of $B$-modules yields an exact sequence (3) of vector spaces, from which we conclude $\left(V / V^{2}\right)^{B}=0$. This completes the induction.

Proposition 4.4. Let $\phi: \mathfrak{g} \rightarrow \mathfrak{g}$ be a B-equivariant $k$-linear map. Then $\phi=\lambda \cdot \mathrm{id}_{\mathfrak{g}}$ for some constant $\lambda \in k$. 
Proof. Since $\phi$ is a $T$-module endomorphism, it is a direct sum of components $\phi_{0} \in \operatorname{End}\left(\mathfrak{g}_{0}\right)$ and $\phi_{\alpha} \in \operatorname{End}\left(\mathfrak{g}_{\alpha}\right)$ for $\alpha \in \Phi$. As $\operatorname{dim} \mathfrak{g}_{\alpha}=1$, we have $\phi_{\alpha}=\lambda_{\alpha} \cdot \operatorname{id}_{\mathfrak{g}_{\alpha}}$ with $\lambda_{\alpha} \in k$. We put $\lambda:=\lambda_{\theta}$ for the highest root $\theta \in \Phi^{+}$.

Suppose that $\alpha=\beta+\gamma$ holds for $\alpha, \beta, \gamma \in \Phi^{+}$, and let $n \geq 1$ be maximal such that $\beta_{0}:=\alpha-n \gamma \in \Phi^{+}$. The action of $U_{\gamma}$ on $\mathfrak{g}$ satisfies

$$
u_{\gamma} \cdot e_{\beta_{0}}=e_{\beta_{0}}+\sum_{m \geq 1} \pm e_{\beta_{0}+m \gamma}
$$

according to [7, p. 64], with the convention $e_{\psi}=0$ for $\psi \notin \Phi$. Comparing eigenvalues of $\phi$ on both sides, we conclude $\lambda_{\beta_{0}}=\lambda_{\beta_{0}+\gamma}=\ldots$, and in particular $\lambda_{\beta}=\lambda_{\alpha}$. By symmetry, we also have $\lambda_{\gamma}=\lambda_{\alpha}$.

Writing $\alpha$ as a sum of simple roots $\alpha_{i_{r}}$, an iteration of this argument shows $\lambda_{\alpha_{i_{r}}}=\lambda_{\alpha}$ for all $r$. All simple roots appear in the highest root, so we get in particular $\lambda_{\alpha_{i}}=\lambda$ for all $i$, and hence $\lambda_{\alpha}=\lambda$ for all $\alpha \in \Phi^{+}$.

For every $\alpha \in \Phi^{+}$, the formulas in [7, p. 64] contain in particular

$$
u_{\alpha} \cdot e_{-\alpha}=e_{-\alpha}+\left[e_{\alpha}, e_{-\alpha}\right]-e_{\alpha} .
$$

Comparing the effect of $\phi$ on both sides shows $\lambda_{-\alpha}=\lambda_{\alpha}=\lambda$.

The map $\mathfrak{g}_{0} \rightarrow \oplus_{i} \mathfrak{g}_{\alpha_{i}}, h \mapsto\left(u_{\alpha_{i}} \cdot h-h\right)_{i}$, is an isomorphism due to the exact sequence (4). Since $\phi$ is $B$-equivariant, it commutes with this isomorphism. Hence $\phi_{0}=\lambda \cdot \mathrm{id}_{\mathfrak{g}_{0}}$ follows; thus $\phi=\lambda \cdot \mathrm{id}_{\mathfrak{g}}$.

Corollary 4.5. The $B$-module $V:=\operatorname{End}(\mathfrak{g}) / k \cdot$ id is a successive extension of $B$-modules of the form

- $\ell_{\alpha}$ for some root $\alpha \in \Phi$, or

- $\ell_{\alpha-\beta}$ for some pair of different roots $\alpha \neq \beta \in \Phi$, or

- $\wp_{i}$ for some simple root $\alpha_{i}$, or

- $\wp_{i}^{2}$ for some simple root $\alpha_{i}$ in the case $\operatorname{char}(k)=2$, or

- $\wp_{1}^{3}$ in the case $\operatorname{char}(k)=3$ and $\Phi \cong G_{2}$.

Proof. We use the short exact sequence of $B$-modules

$$
0 \longrightarrow \ell_{0} \cong k \cdot \mathrm{id} \longrightarrow \operatorname{End}(\mathfrak{g}) \longrightarrow V \longrightarrow 0
$$

Proposition 4.4 states $\operatorname{End}(\mathfrak{g})^{B}=k \cdot$ id. Therefore, the associated exact sequence (3) of vector spaces shows $V^{B}=0$. Hence Corollary 4.3 applies, so $V$ is a successive extension of some $\wp_{i}^{q}$ and some $\ell_{\chi}$ for nontrivial $\chi$.

Here $\ell_{\chi}$ can only appear if the $T$-eigenspace $V_{\chi}$ is nonzero, or in other words if $\chi$ is a root or a difference of roots. Similarly, $\wp_{i}^{q}$ can only appear if $q \alpha_{i}$ is a root or a difference of roots. According to the classification of root systems, the latter happens only if $q \leq 2$, or if $q=3$ and $\Phi \cong G_{2}$ and $\alpha_{i}=\alpha_{1}$ is short.

\section{Proof of Theorem 2.5}

Let $G$ be reductive, with center $Z$, and let $\pi: G \rightarrow G / Z$ be the projection. For each principal $G$-bundle $E$ with induced principal $(G / Z)$-bundle $\pi_{*} E$, we have $\operatorname{Aut}(E) / Z \subseteq \operatorname{Aut}\left(\pi_{*} E\right)$. Thus $\mathcal{M}_{G}^{\mathrm{rs}}$ contains the inverse image of $\mathcal{M}_{G / Z}^{\mathrm{rs}}$ under the 1-morphism $\pi_{*}: \mathcal{M}_{G} \rightarrow \mathcal{M}_{G / Z}$.

As this $\pi_{*}$ is flat according to [3, Lemma 2.2.2], it suffices to prove the theorem for $G / Z$ instead of $G$. Thus we can assume without loss of generality that $G$ is of adjoint type, and also that $G$ is simple. 
Let $E$ be a principal $G$-bundle on $C$. If its adjoint vector bundle $\operatorname{ad}(E)=$ $E \times{ }^{G} \mathfrak{g}$ has only scalar endomorphisms, then $\operatorname{Aut}(E)$ is trivial. This shows that the complement of $\mathcal{M}_{G}^{\mathrm{rs}}$ in $\mathcal{M}_{G}^{\mathrm{s}}$ is contained in the Brill-Noether locus $\mathcal{W}_{\text {End }(\mathfrak{g}) / k \text {.id }} \subseteq$ $\mathcal{M}_{G}$. Hence Theorem 2.5 is a consequence of the following proposition.

Proposition 5.1. Let $G$ be simple of adjoint type. Let $d \in \pi_{1}(G)$ be given.

i) If $g \geq 3$, then the complement of $\mathcal{W}_{\operatorname{End}(\mathfrak{g}) / k \text {.id }}$ in $\mathcal{M}_{G}^{d}$ is non-empty.

ii) If $g \geq 4$, then $\mathcal{W}_{\operatorname{End}(\mathfrak{g}) / k \cdot \text { id }}$ has codimension $\geq 2$ in $\mathcal{M}_{G}^{d}$.

In order to prove this proposition, we use the notation of the previous section. So $T \subseteq B \subseteq G$ is a maximal torus in a Borel subgroup, and

$$
\alpha_{1}, \ldots, \alpha_{l} \in \Phi^{+} \subseteq \Phi \subseteq \operatorname{Hom}\left(T, \mathbb{G}_{\mathrm{m}}\right)
$$

are the simple roots, the positive roots, and all roots of $G$, respectively. If $G$ is of type $G_{2}$, then $\alpha_{1}$ is the short simple root by Convention 4.1.

An element $\delta \in \pi_{1}(B)=\operatorname{Hom}\left(\mathbb{G}_{\mathrm{m}}, T\right)$ is called minuscule if $\delta \neq 0$, and $\langle\alpha, \delta\rangle \in$ $\{0,1\}$ holds for all positive roots $\alpha \in \Phi^{+}$of $G$ with respect to $B$. Every nonzero element $d \in \pi_{1}(G)$ has a unique minuscule lift $\delta \in \pi_{1}(B)$ according to $[6$, Chapitre VIII, §7, Proposition 8].

Lemma 5.2. Suppose that $\delta \in \pi_{1}(B)$ is zero, or that $-\delta$ is minuscule.

i) If $g \geq 3$, then the complement of $\mathcal{W}_{\operatorname{End}(\mathfrak{g}) / k \text {.id }}$ in $\mathcal{M}_{B}^{\delta}$ is non-empty.

ii) If $g \geq 4$, and $\mathcal{W}_{\operatorname{End}(\mathfrak{g}) / k \cdot \text { id }}$ contains a prime divisor $D \subseteq \mathcal{M}_{B}^{\delta}$, then $\Phi \cong G_{2}$, $\delta=0$, and $D=\left(\alpha_{1}\right)_{*}^{-1}\left(D^{\prime}\right)$ for a prime divisor $D^{\prime} \subseteq \mathcal{M}_{\mathbb{G}_{\mathrm{m}}}^{0}$.

Proof. Note that $\mathcal{W}_{V} \subseteq \mathcal{W}_{V^{\prime}} \cup \mathcal{W}_{V^{\prime \prime}}$ for every short exact sequence

$$
0 \longrightarrow V^{\prime} \longrightarrow V \longrightarrow V^{\prime \prime} \longrightarrow 0
$$

of $B$-modules. Using Corollary 4.5 , we may thus replace the $B$-module $\operatorname{End}(\mathfrak{g}) / k$. id by the 1 - and 2-dimensional $B$-modules $V$ listed there.

The cases $V=\ell_{\alpha}$ and $V=\ell_{\alpha-\beta}$ follow from Lemma 3.1. It remains to treat the cases $V=\wp_{i}, V=\wp_{i}^{2}$, and $V=\wp_{1}^{3}$.

The exact sequence (4) shows that the kernel of $\pi_{i}: B \rightarrow \mathbb{G}_{a} \rtimes \mathbb{G}_{\mathrm{m}}$ is smooth. So the induced map $\left(\pi_{i}\right)_{*}: \mathcal{M}_{B} \rightarrow \mathcal{M}_{\mathbb{G}_{a} \rtimes \mathbb{G}_{\mathrm{m}}}$ is also smooth, by deformation theory. Now use Corollary 3.3 and Lemma 3.4 .

Proof of Proposition [5.1, The claim 5.1] i follows from Lemma 5.2]i.

Now suppose $g \geq 4$, and that $\mathcal{W}_{\operatorname{End}(\mathfrak{g}) / k \text {.id }}$ contains a prime divisor $D \subseteq \mathcal{M}_{G}^{d}$. Then its ideal sheaf $\mathcal{O}(-D)$ is a line bundle, since $\mathcal{M}_{G}^{d}$ is smooth. This line bundle is nontrivial because $\mathrm{H}^{0}\left(\mathcal{M}_{G}^{d}, \mathcal{O}\right)=k$, cf. for example [3, Theorem 5.3.1.i].

For any lift $\delta \in \operatorname{Hom}\left(\mathbb{G}_{\mathrm{m}}, T\right)$ of $d$, the pullback of $\mathcal{O}(-D)$ to $\mathcal{M}_{T}^{\delta}$ is still nontrivial, due to [3, Lemma 5.2.6 and Theorem 5.3.1.iv]. In particular, the pullback of $\mathcal{O}(-D)$ to $\mathcal{M}_{B}^{\delta}$ is also nontrivial. Thus the inverse image $\mathcal{W}_{\text {End }(\mathfrak{g}) / k \text {.id }} \subseteq \mathcal{M}_{B}$ contains a prime divisor in $\mathcal{M}_{B}^{\delta}$.

Assuming moreover that $G$ is of type $G_{2}$, we have $\pi_{1}(G)=0$, so $d=0$, and $T=\operatorname{ker}\left(\alpha_{1}\right) \times \operatorname{ker}\left(\alpha_{2}\right)$. The pullback of $\mathcal{O}(-D)$ to $\mathcal{M}_{\operatorname{ker}\left(\alpha_{1}\right)}^{0}$ is nontrivial by [3], Proposition 4.4.7.iii]. Thus $\mathcal{W}_{\operatorname{End}(\mathfrak{g}) / k \text {.id }} \subseteq \mathcal{M}_{B}$ contains a prime divisor in $\mathcal{M}_{B}^{0}$ that is no pullback along $\alpha_{1}$.

These conclusions contradict Lemma 5.2. The claim 5.1,ii follows. 


\section{The obstruction against Poincaré families}

Let the smooth connected group $G$ be reductive, and consider $d \in \pi_{1}(G)$.

Definition 6.1. A Poincaré family for an open subscheme $U \subseteq \mathfrak{M}_{G}^{d, \text { rs }}$ is a principal $G$-bundle $\mathcal{E}$ on $C \times U$ such that for every point $x \in U$, the corresponding isomorphism class of stable $G$-bundles contains $\left.\mathcal{E}\right|_{C \times\{x\}}$.

Let $Z \subseteq G$ denote the scheme-theoretic center. The coarse moduli map

$$
\pi: \mathcal{M}_{G}^{d, \mathrm{rs}} \longrightarrow \mathfrak{M}_{G}^{d, \mathrm{rs}}
$$

is a gerbe with band $Z$ for the étale topology; local sections of $\pi$ are given by the étale slices in the proof of Proposition 2.3. Let

$$
\psi_{G}^{d} \in \mathrm{H}_{\mathrm{et}}^{2}\left(\mathfrak{M}_{G}^{d, \mathrm{rs}}, Z\right)
$$

be the cohomology class corresponding to the gerbe $\pi$, according to [1]. Note that a Poincaré family for $U$ is a section of the gerbe $\pi$ over $U$. Thus $\psi_{G}^{d}$ vanishes if and only if there is a Poincaré family for $\mathfrak{M}_{G}^{d, r s}$.

Now suppose $\mathfrak{M}_{G}^{d, r s} \neq \emptyset$. Slightly abusing notation, we also denote by $\psi_{G}^{d}$ its restriction to the generic point $\eta_{\mathfrak{M}_{G}^{d}} \in \mathfrak{M}_{G}^{d, r s}$. This restriction

$$
\psi_{G}^{d} \in \mathrm{H}_{\text {ét }}^{2}\left(\eta_{\mathfrak{M}_{G}^{d}}, Z\right)
$$

vanishes if and only if there is a Poincaré family for some open subscheme $\emptyset \neq U \subseteq$ $\mathfrak{M}_{G}^{d, \mathrm{rs}}$. In this section, we determine the order of these obstruction classes. The result is given in terms of the root system of $G$.

Let $G$ for the moment be of adjoint type. Choose a maximal torus $T \subseteq G$. Let $\Lambda_{\text {coroots }} \subseteq \Lambda_{T}:=\operatorname{Hom}\left(\mathbb{G}_{\mathrm{m}}, T\right)$ denote the subgroup generated by the coroots of $G$. The Weyl group $W$ of $(G, T)$ acts on $\Lambda_{T}$. This action preserves the subgroup $\Lambda_{\text {coroots }}$, and the induced action on the quotient group $\Lambda_{T} / \Lambda_{\text {coroots }}=\pi_{1}(G)$ is trivial.

Lemma 6.2. Given an even $W$-invariant symmetric bilinear form

$$
\Lambda_{\text {coroots }} \times \Lambda_{\text {coroots }} \longrightarrow \mathbb{Z}
$$

its bilinear extension $\Lambda_{T} \times \Lambda_{T} \rightarrow \mathbb{Q}$ descends to a symmetric bilinear map

$$
\pi_{1}(G) \times \pi_{1}(G) \longrightarrow \mathbb{Q} / \mathbb{Z}
$$

Proof. Let $b: \Lambda_{\text {coroots }} \times \Lambda_{\text {coroots }} \rightarrow \mathbb{Z}$ be $W$-invariant, symmetric, bilinear, and even in the sense that $b(\lambda, \lambda)$ is even for all $\lambda \in \Lambda_{\text {coroots. }}$ Given a root $\alpha: \Lambda_{T} \rightarrow \mathbb{Z}$ of $G$, with corresponding coroot $\alpha^{\vee} \in \Lambda_{T}$, the formula

$$
b\left(\lambda, \alpha^{\vee}\right)=\alpha(\lambda) \cdot b\left(\alpha^{\vee}, \alpha^{\vee}\right) / 2
$$

holds for all $\lambda \in \Lambda_{\text {coroots }}$, according to [5, Chapitre VI, §1, Lemme 2]. In particular, the map $b\left(\_, \alpha^{\vee}\right): \Lambda_{\text {coroots }} \rightarrow \mathbb{Z}$ is an integral multiple of $\alpha$, and hence extends to a linear map $\Lambda_{T} \rightarrow \mathbb{Z}$. Thus the bilinear extension $\Lambda_{T} \times \Lambda_{T} \rightarrow \mathbb{Q}$ of $b$ is integral on $\Lambda_{T} \times \Lambda_{\text {coroots }}$, and also on $\Lambda_{\text {coroots }} \times \Lambda_{T}$ by symmetry. Hence the composition $\Lambda_{T} \times \Lambda_{T} \rightarrow \mathbb{Q} \rightarrow \mathbb{Q} / \mathbb{Z}$ descends to a bilinear map $\pi_{1}(G) \times \pi_{1}(G) \rightarrow \mathbb{Q} / \mathbb{Z}$.

Definition 6.3. Suppose that $G$ is of adjoint type. We denote by

$$
\Psi(G) \subseteq \operatorname{Hom}\left(\pi_{1}(G) \otimes \pi_{1}(G), \mathbb{Q} / Z\right)
$$




\begin{tabular}{|c|c|c|c|c|}
\hline type of $G$ & $\pi_{1}(G)$ & $\Psi(G)$ & generator of $\Psi(G)$ & \\
\hline$A_{l}, l \geq 1$ & $\mathbb{Z} / l+1$ & $\mathbb{Z} / l+1$ & mult: $\mathbb{Z} / l+1 \otimes \mathbb{Z} / l+1 \longrightarrow \mathbb{Z} / l+1$ & \\
\hline$B_{l}, l \geq 2$ & $\mathbb{Z} / 2$ & 0 & - & \\
\hline$C_{l}, l \geq 3$ & $\mathbb{Z} / 2$ & $\begin{array}{l}\mathbb{Z} / 2 \\
0\end{array}$ & mult: $\begin{array}{c}\mathbb{Z} / 2 \otimes \mathbb{Z} / 2 \longrightarrow{ }^{\mathbb{Z}} / 2 \\
-\end{array}$ & $\begin{array}{l}l \text { odd } \\
l \text { even }\end{array}$ \\
\hline$D_{l}, l \geq 4$ & $\begin{array}{l}\mathbb{Z} / 4 \\
(\mathbb{Z} / 2)^{2}\end{array}$ & $\begin{array}{l}\mathbb{Z} / 4 \\
\mathbb{Z} / 2\end{array}$ & $\begin{array}{c}\text { mult: } \mathbb{Z} / 4 \otimes \mathbb{Z} / 4 \longrightarrow \mathbb{Z} / 4 \\
\left(\begin{array}{ll}0 & 1 \\
1 & 0\end{array}\right):(\mathbb{Z} / 2)^{2} \otimes(\mathbb{Z} / 2)^{2} \longrightarrow \mathbb{Z} / 2 \\
\left(\begin{array}{ll}1 & 0 \\
0 & 1\end{array}\right):(\mathbb{Z} / 2)^{2} \otimes(\mathbb{Z} / 2)^{2} \longrightarrow \mathbb{Z} / 2\end{array}$ & $\begin{array}{c}l \text { odd } \\
l \in 4 \mathbb{Z} \\
l \in 4 \mathbb{Z}+2\end{array}$ \\
\hline$E_{6}$ & $\mathbb{Z} / 3$ & $\mathbb{Z} / 3$ & mult: $\mathbb{Z} / 3 \otimes \mathbb{Z} / 3 \longrightarrow \mathbb{Z} / 3$ & \\
\hline$E_{7}$ & $\mathbb{Z} / 2$ & $\mathbb{Z} / 2$ & mult: $\mathbb{Z} / 2 \otimes \mathbb{Z} / 2 \longrightarrow \mathbb{Z} / 2$ & \\
\hline$E_{8}$ & 0 & 0 & - & \\
\hline$F_{4}$ & 0 & 0 & - & \\
\hline$G_{2}$ & 0 & 0 & - & \\
\hline
\end{tabular}

TABLE 1. The abelian group $\Psi(G)$ for simple $G$ of adjoint type.

(To obtain the required maps to $\mathbb{Q} / \mathbb{Z}$, embed $\mathbb{Z} / n$ into $\mathbb{Q} / \mathbb{Z}$ ).

the abelian group of all bilinear maps $b: \pi_{1}(G) \times \pi_{1}(G) \rightarrow \mathbb{Q} / \mathbb{Z}$ that come from even $W$-invariant symmetric bilinear forms $\Lambda_{\text {coroots }} \times \Lambda_{\text {coroots }} \rightarrow \mathbb{Z}$.

Note that $\Psi(G)$ is determined by the root system of $G$. If $G=G_{1} \times G_{2}$, then $\Psi(G)=\Psi\left(G_{1}\right) \oplus \Psi\left(G_{2}\right)$. If $G$ is a simple group, then the abelian group $\Psi(G)$ is cyclic, and a generator is given by Table 1 .

Now return to the general case where $G$ is reductive. Let $Z^{0}$ be the (reduced) identity component in the center $Z$ of $G$. The central isogenies

$$
G^{\prime}:=[G, G] \rightarrow \bar{G}:=G / Z^{0} \rightarrow G^{\text {ad }}:=G / Z
$$

correspond to subgroups $\pi_{1}\left(G^{\prime}\right) \subseteq \pi_{1}(\bar{G}) \subseteq \pi_{1}\left(G^{\text {ad }}\right)$. If $G$ is semisimple, then $G^{\prime}=\bar{G}=G$, so we just have one subgroup $\pi_{1}(G) \subseteq \pi_{1}\left(G^{\text {ad }}\right)$.

Definition 6.4. For reductive $G$, we denote by $\Psi^{\prime}(G) \subseteq \Psi\left(G^{\text {ad }}\right)$ the subgroup of all elements $b: \pi_{1}\left(G^{\text {ad }}\right) \times \pi_{1}\left(G^{\text {ad }}\right) \rightarrow \mathbb{Q} / \mathbb{Z}$ in $\Psi\left(G^{\text {ad }}\right)$ with

$$
b\left(\pi_{1}(\bar{G}) \times \pi_{1}\left(G^{\prime}\right)\right)=0 .
$$

Given an element $d \in \pi_{1}(G)$ with image $\bar{d} \in \pi_{1}(\bar{G})$, we denote by

$$
\mathrm{ev}_{G}^{d}: \Psi^{\prime}(G) \longrightarrow \operatorname{Hom}\left(\frac{\pi_{1}\left(G^{\mathrm{ad}}\right)}{\pi_{1}\left(G^{\prime}\right)}, \mathbb{Q} / \mathbb{Z}\right)
$$

the evaluation map that sends $b$ to $b(\bar{d},-): \pi_{1}\left(G^{\text {ad }}\right) \rightarrow \mathbb{Q} / Z$.

Remark 6.5. This finite abelian group $\operatorname{Hom}\left(\frac{\pi_{1}\left(G^{\text {ad }}\right)}{\pi_{1}\left(G^{\prime}\right)}, \mathbb{Q} / \mathbb{Z}\right)$ can be identified with the character group $\operatorname{Hom}\left(Z^{\prime}, \mathbb{G}_{\mathrm{m}}\right)$ of the center $Z^{\prime} \subseteq G^{\prime}$; cf. the equations (7) in the proof of Proposition 7.2 below. 
Theorem 6.6. Assume $g \geq 3$. Let $G$ be reductive, with scheme-theoretic center $Z$. The order of $\psi_{G}^{d}$ in $\mathrm{H}_{\text {ét }}^{2}\left(\eta_{\mathfrak{M}_{G}^{d}}, Z\right)$ is the exponent of the finite abelian group coker $\left(\operatorname{ev}_{G}^{d}\right)$ in Definition 6.4.

Corollary 6.7. Assume $g \geq 3$. There is a Poincaré family for some non-empty open subscheme $U \subseteq \mathfrak{M}_{G}^{d}$ if and only if the evaluation map $\mathrm{ev}_{G}^{d}$ in Definition 6.4 is surjective.

Theorem 6.8. Assume $g \geq 4$. The order of $\psi_{G}^{d}$ in $\mathrm{H}_{\text {ét }}^{2}\left(\mathfrak{M}_{G}^{d, \mathrm{rs}}, Z\right)$ is the least common multiple of its order in $\mathrm{H}_{\mathrm{et}}^{2}\left(\eta_{\mathfrak{M}_{G}^{d}}, Z\right)$ and the exponent of the finite abelian group $\operatorname{Hom}\left(Z / Z^{0}, \mathbb{G}_{\mathrm{m}}\right)$.

Corollary 6.9. Assume $g \geq 4$, and that there is a Poincaré family for some nonempty open subscheme $U \subseteq \mathfrak{M}_{G}^{d}$. Then there is a Poincaré family for $\mathfrak{M}_{G}^{d, \mathrm{rs}}$ if and only if $Z$ is a torus.

Theorem 6.6 and Theorem 6.8 are proved in the next section.

Remark 6.10. The assumption $g \geq 3$ (respectively, $g \geq 4$ ) is needed only to ensure that $\mathcal{M}_{G}^{d, \text { rs }}$ is non-empty (respectively, that its complement has codimension $\geq 2$ in $\left.\mathcal{M}_{G}^{d}\right)$. In the case $\operatorname{char}(k)=0$, these statements about $\mathcal{M}_{G}^{d, \mathrm{rs}}$ are proved also for smaller $g$ in [10, Theorem II.6]. In this case, Theorem 6.6 and its Corollary 6.7 follow also for $g=2$, while Theorem 6.8 and its Corollary 6.9 follow also for $g=3$, and even for $g=2$ unless there is a nontrivial homomorphism $G \rightarrow \mathrm{PGL}_{2}$.

\section{Gerbes with Band $Z$}

We say that an algebraic group $Z$ over $k$ is of multiplicative type if it is of the form $Z \cong \mathbb{G}_{\mathrm{m}}^{r} \times \mu_{n_{1}} \times \cdots \times \mu_{n_{s}}$ with $r, s \geq 0$ and $n_{1}, \ldots, n_{s} \geq 1$.

Let $U$ be an integral scheme of finite type over $k$. Let $\pi: \mathcal{U} \rightarrow U$ be a gerbe with band $Z$ for an algebraic group $Z$ of multiplicative type over $k$. We denote the cohomology class of this gerbe by $\psi_{\mathcal{U}} \in \mathrm{H}_{\text {ét }}^{2}(U, Z)$.

The stack $\mathcal{U}$ is given by a groupoid $\mathcal{U}(S)$ for each $k$-scheme $S$. Since $\pi$ is a gerbe with band $Z$, we have an isomorphism $\iota_{\mathcal{E}}: Z(S) \rightarrow \operatorname{Aut}_{\mathcal{U}(S)}(\mathcal{E})$ for every object $\mathcal{E}$ in $\mathcal{U}(S)$. These data are subject to appropriate compatibility conditions.

Now let $\mathcal{L}$ be a line bundle on $\mathcal{U}$. Recall that $\mathcal{L}$ is given by a functor $\mathcal{L}_{S}$ from $\mathcal{U}(S)$ to the groupoid of line bundles on $S$ for every $k$-scheme $S$. In particular, $\mathcal{L}_{S}$ defines for every object $\mathcal{E}$ in $\mathcal{U}(S)$ a group homomorphism

$$
\mathcal{L}_{S, \mathcal{E}}: \operatorname{Aut}_{\mathcal{U}(S)}(\mathcal{E}) \longrightarrow \operatorname{Aut}_{\mathcal{O}_{S}}\left(\mathcal{L}_{S}(\mathcal{E})\right)=\Gamma\left(S, \mathcal{O}_{S}^{*}\right) .
$$

The compatibility conditions ensure that the compositions

$$
Z(S) \stackrel{\iota_{\mathcal{E}}}{\longrightarrow} \operatorname{Aut}_{\mathcal{U}(S)}(\mathcal{E}) \stackrel{\mathcal{L}_{S, \mathcal{E}}}{\longrightarrow} \Gamma\left(S, \mathcal{O}_{S}^{*}\right)
$$

define a 1-morphism $Z \times \mathcal{U} \rightarrow \mathbb{G}_{\mathrm{m}} \times \mathcal{U}$ over $\mathcal{U}$. Because $\mathcal{U}$ is connected and $\operatorname{Hom}\left(Z, \mathbb{G}_{\mathrm{m}}\right)$ is discrete, this 1-morphism is the pullback of some character $\chi: Z \rightarrow$ $\mathbb{G}_{\mathrm{m}}$. We call $\chi$ the weight of $\mathcal{L}$. Sending each line bundle $\mathcal{L}$ on $\mathcal{U}$ to its weight $\chi$ defines a group homomorphism

$$
\text { wt }: \operatorname{Pic}(\mathcal{U}) \longrightarrow \operatorname{Hom}\left(Z, \mathbb{G}_{\mathrm{m}}\right) \text {. }
$$

If $\mathcal{L}$ has trivial weight, then it descend to $U$, so we have an exact sequence

$$
0 \longrightarrow \operatorname{Pic}(U) \longrightarrow \operatorname{Pic}(\mathcal{U}) \stackrel{\text { wt }}{\longrightarrow} \operatorname{Hom}\left(Z, \mathbb{G}_{\mathrm{m}}\right) .
$$


Example 7.1. Let $\emptyset \neq \mathcal{U} \subseteq \mathcal{M}_{G}^{d}$ be an open substack, with $G$ reductive. Given a $k$-scheme $S$, the objects in $\mathcal{U}(S)$ are principal $G$-bundles $\mathcal{E}$ on $C \times S$. The center $Z \subseteq G$ acts by automorphisms on every principal $G$-bundle. Thus we obtain a homomorphism $\iota_{\mathcal{E}}: Z(S) \rightarrow \operatorname{Aut}_{\mathcal{U}(S)}(\mathcal{E})$ for every object $\mathcal{E}$ in $\mathcal{U}(S)$.

Suppose $\mathcal{U} \subseteq \mathcal{M}_{G}^{d, \mathrm{rs}}$, and let $U \subseteq \mathfrak{M}_{G}^{d, \mathrm{rs}}$ be the corresponding open subscheme. Then all $\iota_{\mathcal{E}}$ are isomorphisms, and they turn the coarse moduli map $\pi: \mathcal{U} \rightarrow U$ into a gerbe with band $Z$. In particular, the action of $Z$ on fibers of line bundles $\mathcal{L}$ on $\mathcal{U}$ defines a group homomorphism

$$
\mathrm{wt}=\mathrm{wt}_{G}^{d}: \operatorname{Pic}(\mathcal{U}) \longrightarrow \operatorname{Hom}\left(Z, \mathbb{G}_{\mathrm{m}}\right) .
$$

However, the definition of $\operatorname{wt}(\mathcal{L})$ used only the homomorphisms $\iota_{\mathcal{E}}$, not the fact that they are isomorphisms. Thus we can drop the assumption $\mathcal{U} \subseteq \mathcal{M}_{G}^{d, \mathrm{rs}}$, and the same construction yields a group homomorphism

$$
\mathrm{wt}=\mathrm{wt}_{G}^{d}: \operatorname{Pic}(\mathcal{U}) \longrightarrow \operatorname{Hom}\left(Z, \mathbb{G}_{\mathrm{m}}\right)
$$

for every non-empty open substack $\mathcal{U} \subseteq \mathcal{M}_{G}^{d}$.

Proposition 7.2. Given $G$ reductive and $d \in \pi_{1}(G)$, consider the map

$$
\mathrm{wt}=\mathrm{wt}_{G}^{d}: \operatorname{Pic}\left(\mathcal{M}_{G}^{d}\right) \longrightarrow \operatorname{Hom}\left(Z, \mathbb{G}_{\mathrm{m}}\right) \text {. }
$$

i) The kernel of $\mathrm{wt}_{G}^{d}$ contains the torsion in $\operatorname{Pic}\left(\mathcal{M}_{G}^{d}\right)$.

ii) The cokernel of $\mathrm{wt}_{G}^{d}$ is isomorphic to the cokernel of the map $\mathrm{ev}_{G}^{d}$ in Definition 6.4.

Proof. In [3, Definition 5.2.1], we have defined a finitely generated free abelian group $\mathrm{NS}\left(\mathcal{M}_{G}^{d}\right)$ in terms of the root system of $G$. Instead of repeating the definition, we just recall the properties that we need here:

(a) There is a canonical epimorphism $c_{G}: \operatorname{Pic}\left(\mathcal{M}_{G}^{d}\right) \rightarrow \mathrm{NS}\left(\mathcal{M}_{G}^{d}\right)$.

(b) Every homomorphism of reductive groups $\varphi: G \rightarrow H$ induces a homomorphism of abelian groups $\varphi^{\mathrm{NS}, d}$ such that the diagram

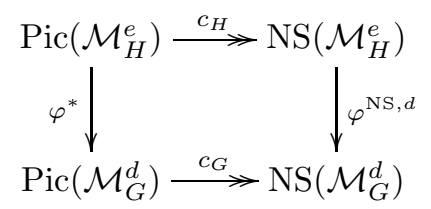

commutes, with $e:=\varphi_{*}(d) \in \pi_{1}(H)$.

(c) If $G=T$ is a torus, then $\operatorname{NS}\left(\mathcal{M}_{T}^{d}\right)=\operatorname{Hom}\left(T, \mathbb{G}_{\mathrm{m}}\right) \oplus \mathrm{NS}\left(\mathfrak{M}_{T}^{0}\right)$, and the weight map $\operatorname{wt}_{T}^{d}: \operatorname{Pic}\left(\mathcal{M}_{T}^{d}\right) \rightarrow \operatorname{Hom}\left(T, \mathbb{G}_{\mathrm{m}}\right)$ is the composition

$$
\operatorname{Pic}\left(\mathcal{M}_{T}^{d}\right) \stackrel{c_{T}}{\longrightarrow} \operatorname{NS}\left(\mathcal{M}_{T}^{d}\right) \stackrel{\mathrm{pr}_{1}}{\longrightarrow} \operatorname{Hom}\left(T, \mathbb{G}_{\mathrm{m}}\right) .
$$

(d) Choose a maximal torus $T \subseteq G$. In the notation of Definition 6.4 let $T^{\prime} \rightarrow \bar{T} \rightarrow T^{\text {ad }}$ be the induced tori in $G^{\prime} \rightarrow \bar{G} \rightarrow G^{\text {ad }}$. Their lattices

$\Lambda_{T^{\prime}}:=\operatorname{Hom}\left(\mathbb{G}_{\mathrm{m}}, T^{\prime}\right) \subseteq \Lambda_{\bar{T}}:=\operatorname{Hom}\left(\mathbb{G}_{\mathrm{m}}, \bar{T}\right) \subseteq \Lambda_{T^{\mathrm{ad}}}:=\operatorname{Hom}\left(\mathbb{G}_{\mathrm{m}}, T^{\mathrm{ad}}\right)$

all contain $\Lambda_{\text {coroots }}$. Let $\widetilde{\Psi}(G)$ denote the group of all bilinear maps

$$
b: \Lambda_{\bar{T}} \times \Lambda_{T^{\prime}} \longrightarrow \mathbb{Z}
$$


whose restriction $\Lambda_{\text {coroots }} \times \Lambda_{\text {coroots }} \rightarrow \mathbb{Z}$ is $W$-invariant, symmetric, and even. Then one has a canonical exact sequence

$$
0 \longrightarrow \mathrm{NS}\left(\mathcal{M}_{G / G^{\prime}}^{e}\right) \stackrel{\pi^{\mathrm{NS}, d}}{\longrightarrow} \mathrm{NS}\left(\mathcal{M}_{G}^{d}\right) \stackrel{\mathrm{pr}_{2}}{\longrightarrow} \widetilde{\Psi}(G) \longrightarrow 0
$$

where $\pi: G \rightarrow G / G^{\prime}$ is the projection, and $e:=\pi_{*}(d) \in \pi_{1}\left(G / G^{\prime}\right)$.

(e) Let $\iota: T \hookrightarrow G$ be the inclusion. Choose a lift $\delta \in \Lambda_{T}$ of $d \in \pi_{1}(G)$, and let $\bar{\delta} \in \Lambda_{\bar{T}}$ be the image of $\delta$. Then the following diagram commutes:

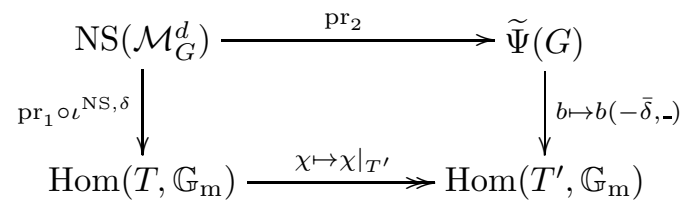

These properties are contained in Proposition 5.2.11 and Theorem 5.3.1 of [3]; see also its Subsection 3.2 for (c), and its Definition 5.2.5 for (e).

The inclusion $\iota: T \hookrightarrow G$ of a maximal torus and the maximal commutative quotient $\pi: G \rightarrow G / G^{\prime}$ induce a commutative diagram

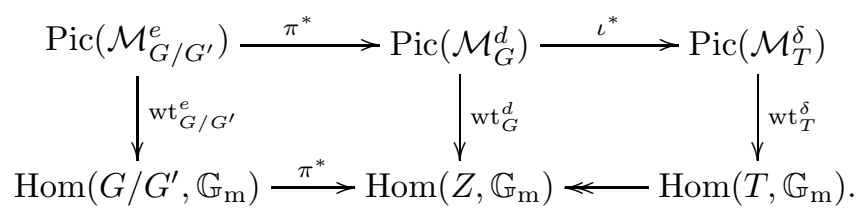

In particular, the map $\mathrm{wt}_{G}^{d}$ factors through the torsionfree abelian group $\operatorname{Hom}\left(T, \mathbb{G}_{\mathrm{m}}\right)$; this proves part (i).

Let $Z^{\prime}$ denote the scheme-theoretic center of $G^{\prime}$; then $Z / Z^{\prime} \cong G / G^{\prime}$. In this diagram (5), the map $\mathrm{wt}_{G / G^{\prime}}^{e}$ is surjective by property (c), so the image of $\mathrm{wt}_{G}^{d}$ contains the image $\operatorname{Hom}\left(Z / Z^{\prime}, \mathbb{G}_{\mathrm{m}}\right)$ of $\pi^{*}$; consequently, the cokernel of $\mathrm{wt}_{G}^{d}$ is isomorphic to the cokernel of the composition

$$
\operatorname{Pic}\left(\mathcal{M}_{G}^{d}\right) \stackrel{\iota^{*}}{\longrightarrow} \operatorname{Pic}\left(\mathcal{M}_{T}^{\delta}\right) \stackrel{\mathrm{wt}_{T}^{\delta}}{\longrightarrow} \operatorname{Hom}\left(T, \mathbb{G}_{\mathrm{m}}\right) \rightarrow \operatorname{Hom}\left(Z^{\prime}, \mathbb{G}_{\mathrm{m}}\right) .
$$

Using the canonical identification

$$
\operatorname{Hom}\left(Z^{\prime}, \mathbb{G}_{\mathrm{m}}\right)=\frac{\operatorname{Hom}\left(\Lambda_{T^{\prime}}, \mathbb{Z}\right)}{\operatorname{Hom}\left(\Lambda_{T^{\mathrm{ad}}}, \mathbb{Z}\right)}=\operatorname{Hom}\left(\frac{\Lambda_{T^{\mathrm{ad}}}}{\Lambda_{T^{\prime}}}, \frac{\mathbb{Q}}{\mathbb{Z}}\right)=\operatorname{Hom}\left(\frac{\pi_{1}\left(G^{\mathrm{ad}}\right)}{\pi_{1}\left(G^{\prime}\right)}, \frac{\mathbb{Q}}{\mathbb{Z}}\right),
$$

the above properties (a) - (e) yield a commutative diagram

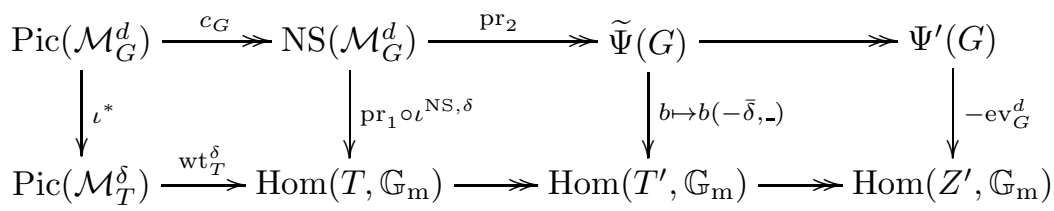

where the unlabeled arrows are the obvious canonical surjections. Because the three maps in the top row of this diagram are surjective, the cokernel of the composition (6) is isomorphic to the cokernel of $\operatorname{ev}_{G}^{d}$. 
Lemma 7.3. Let $\mathcal{M}$ be an Artin stack which is locally of finite type and smooth over $k$. Let $\mathcal{U} \subseteq \mathcal{M}$ be an open substack.

i) The restriction map $\operatorname{Pic}(\mathcal{M}) \rightarrow \operatorname{Pic}(\mathcal{U})$ is surjective.

ii) If $\mathcal{M} \backslash \mathcal{U}$ has codimension $\geq 2$ in $\mathcal{M}$, then $\operatorname{Pic}(\mathcal{M})=\operatorname{Pic}(\mathcal{U})$.

Proof. i) Suppose $\mathcal{U} \subsetneq \mathcal{M}$, and let $\mathcal{L}$ be a line bundle on $\mathcal{U}$. Using Zorn's lemma, it suffices to extend $\mathcal{L}$ to a line bundle on $\mathcal{M}^{\prime}$ for some open substack $\mathcal{U} \subsetneq \mathcal{M}^{\prime} \subseteq \mathcal{M}$. We can choose a quasi-compact open substack $\mathcal{U}^{\prime} \subseteq \mathcal{M}$ not contained in $\mathcal{U}$, and take $\mathcal{M}^{\prime}:=\mathcal{U} \cup \mathcal{U}^{\prime}$. To extend $\mathcal{L}$ to $\mathcal{M}^{\prime}$, it suffices to extend $\left.\mathcal{L}\right|_{\mathcal{U} \cap \mathcal{U}^{\prime}}$ to $\mathcal{U}^{\prime}$. Thus we may assume without loss of generality that $\mathcal{M}$ is quasi-compact.

Now $\mathcal{M}$ and $\mathcal{U}$ are of finite type over $k$. Then [19, Corollaire 15.5] allows us to extend $\mathcal{L}$ to a coherent sheaf on $\mathcal{M}$, namely to a coherent subsheaf of $j_{*}(\mathcal{L})$, where $j: \mathcal{U} \hookrightarrow \mathcal{M}$ is the inclusion. Using smoothness, the bidual of this coherent sheaf of rank one on $\mathcal{M}$ is the required line bundle extending $\mathcal{L}$.

ii) Suppose that $\mathcal{M} \backslash \mathcal{U}$ has codimension $\geq 2$ in $\mathcal{M}$, and let $\mathcal{L}$ be a line bundle on $\mathcal{M}$. Every section of $\mathcal{L}$ or $\mathcal{L}^{-1}$ over $\mathcal{U}$ extends uniquely to $\mathcal{M}$ by Hartog's theorem. Hence the restriction map $\operatorname{Pic}(\mathcal{M}) \rightarrow \operatorname{Pic}(\mathcal{U})$ is also injective.

Lemma 7.4. Let $\varphi: Z \rightarrow Z^{\prime}$ be a homomorphism of algebraic groups over $k$, with $Z$ and $Z^{\prime}$ of multiplicative type. Given an integral scheme $U$ of finite type over $k$, and a gerbe $\pi: \mathcal{U} \rightarrow U$ with band $Z$, the following conditions are equivalent:

i) The class $\psi_{\mathcal{U}} \in \mathrm{H}_{\text {ét }}^{2}(U, Z)$ is in the kernel of $\mathrm{H}_{\text {ét }}^{2}(U, Z) \stackrel{\varphi_{*}}{\longrightarrow} \mathrm{H}_{\text {ét }}^{2}\left(U, Z^{\prime}\right)$.

ii) There is a group homomorphism $\sigma: \operatorname{Hom}\left(Z^{\prime}, \mathbb{G}_{\mathrm{m}}\right) \rightarrow \operatorname{Pic}(\mathcal{U})$ with

$$
\text { wt } \circ \sigma=\varphi^{*}: \operatorname{Hom}\left(Z^{\prime}, \mathbb{G}_{\mathrm{m}}\right) \longrightarrow \operatorname{Hom}\left(Z, \mathbb{G}_{\mathrm{m}}\right) \text {. }
$$

Proof. Let $\pi^{\prime}: \mathcal{U}^{\prime} \rightarrow U$ be a gerbe with band $Z^{\prime}$, and let $\psi_{\mathcal{U}^{\prime}} \in \mathrm{H}_{\text {êt }}^{2}\left(U, Z^{\prime}\right)$ be its class. According to Proposition IV.3.1.5 and Théorème IV.3.4.2 in [11, the relation $\varphi_{*}\left(\psi_{\mathcal{U}}\right)=\psi_{\mathcal{U}^{\prime}}$ holds if and only if there is a 1-morphism $\mathcal{U} \rightarrow \mathcal{U}^{\prime}$ over $U$ which induces $\varphi$ on automorphism groups.

In particular, $\psi_{\mathcal{U}}$ is in the kernel of $\varphi_{*}$ if and only if there is a 1-morphism $\Sigma: \mathcal{U} \rightarrow B Z^{\prime}$ which induces $\varphi$ on automorphism groups.

Given such a 1-morphism $\Sigma$, the required group homomorphism $\sigma$ is

$$
\sigma:=\Sigma^{*}: \operatorname{Hom}\left(Z^{\prime}, \mathbb{G}_{\mathrm{m}}\right)=\operatorname{Pic}\left(B Z^{\prime}\right) \longrightarrow \operatorname{Pic}(\mathcal{U}) \text {. }
$$

Conversely, let $\sigma: \operatorname{Hom}\left(Z^{\prime}, \mathbb{G}_{\mathrm{m}}\right) \rightarrow \operatorname{Pic}(\mathcal{U})$ be given. Choosing an isomorphism

$$
Z^{\prime} \cong \mathbb{G}_{\mathrm{m}}^{r} \times \mu_{n_{1}} \times \cdots \times \mu_{n_{s}}
$$

let $\chi_{1}, \ldots, \chi_{r}: Z^{\prime} \rightarrow \mathbb{G}_{\mathrm{m}}$ and $\chi_{r+i}: Z^{\prime} \rightarrow \mu_{n_{i}}$ for $i=1, \ldots, s$ be the projections. Their images under $\sigma$ are line bundles $\mathcal{L}_{1}, \ldots, \mathcal{L}_{r+s}$ on $\mathcal{U}$ such that $\mathcal{L}_{r+i}^{\otimes n_{i}}$ is trivial for all $i$.

The tuple $\left(\mathcal{L}_{1}, \ldots, \mathcal{L}_{r}\right)$ defines a 1-morphism $\mathcal{U} \rightarrow B \mathbb{G}_{\mathrm{m}}^{r}$, and $\mathcal{L}_{r+i}$ together with a trivialisation of $\mathcal{L}_{r+i}^{\otimes n_{i}}$ defines a 1-morphism $\mathcal{U} \rightarrow B \mu_{n_{i}}$. Let $\Sigma: \mathcal{U} \rightarrow B Z^{\prime}$ be the product of these 1-morphisms. If wt $\circ \sigma=\varphi^{*}$, then $\Sigma$ induces $\varphi$ on automorphism groups, and $\varphi_{*}\left(\psi_{\mathcal{U}}\right)=0$ follows.

Proof of Theorem 6.6. Consider a positive integer $n \geq 1$.

Suppose $n \cdot \psi_{G}^{d}=0$ generically. Then $n \cdot \psi_{\mathcal{U}}=0 \in \mathrm{H}_{\text {ét }}^{2}(U, Z)$ for some open subscheme $\emptyset \neq U \subseteq \mathfrak{M}_{G}^{d, \mathrm{rs}}$, with $\mathcal{U}:=\pi^{-1}(U) \subseteq \mathcal{M}_{G}^{d, \mathrm{rs}}$. Applying Lemma 7.4 to $n: Z \rightarrow Z$, we get a map

$$
\sigma: \operatorname{Hom}\left(Z, \mathbb{G}_{\mathrm{m}}\right) \longrightarrow \operatorname{Pic}(\mathcal{U})
$$


with $\mathrm{wt}_{G}^{d} \circ \sigma=n$. In particular, the cokernel of

$$
\mathrm{wt}_{G}^{d}: \operatorname{Pic}(\mathcal{U}) \longrightarrow \operatorname{Hom}\left(Z, \mathbb{G}_{\mathrm{m}}\right)
$$

is annihilated by $n$. But $\operatorname{wt}_{G}^{d}: \operatorname{Pic}\left(\mathcal{M}_{G}^{d}\right) \rightarrow \operatorname{Hom}\left(Z, \mathbb{G}_{\mathrm{m}}\right)$ has the same cokernel, because the restriction map $\operatorname{Pic}\left(\mathcal{M}_{G}^{d}\right) \rightarrow \operatorname{Pic}(\mathcal{U})$ is surjective due to Lemma 7.3. i. Using Proposition 7.2 ,ii, it follows that the cokernel of $\operatorname{ev}_{G}^{d}$ is also annihilated by $n$.

Conversely, suppose that $\operatorname{coker}\left(\operatorname{ev}_{G}^{d}\right)$ is annihilated by $n$. We write

$$
\operatorname{Hom}\left(Z, \mathbb{G}_{\mathrm{m}}\right) \cong \mathbb{Z}^{r} \oplus \mathbb{Z} / n_{1} \oplus \cdots \oplus \mathbb{Z} / n_{s},
$$

and let $\chi_{1}, \ldots, \chi_{r+s} \in \operatorname{Hom}\left(Z, \mathbb{G}_{\mathrm{m}}\right)$ be the corresponding generators. Using the assumption, Proposition 7.2 ,ii allows us to find line bundles

$$
\mathcal{L}_{1}, \ldots, \mathcal{L}_{r+s} \in \operatorname{Pic}\left(\mathcal{M}_{G}^{d}\right)
$$

such that $\mathcal{L}_{i}$ has weight $n \chi_{i}$. Then the line bundles

$$
\mathcal{L}_{r+1}^{\otimes n_{1}}, \ldots, \mathcal{L}_{r+s}^{\otimes n_{s}}
$$

all have trivial weight. Hence their restrictions descend to $\mathfrak{M}_{G}^{d, r s}$, and are thus trivial over some open subscheme $\emptyset \neq U \subseteq \mathfrak{M}_{G}^{d, \text { rs }}$. Consequently, the line bundles $\mathcal{L}_{1}, \ldots, \mathcal{L}_{r+s}$ define a group homomorphism

$$
\sigma: \operatorname{Hom}\left(Z, \mathbb{G}_{\mathrm{m}}\right) \longrightarrow \operatorname{Pic}(\mathcal{U}), \quad \mathcal{U}:=\pi^{-1}(U) \subseteq \mathcal{M}_{G}^{d, \mathrm{rs}} .
$$

We have $\mathrm{wt}_{G}^{d} \circ \sigma=n$ by construction. Hence $n \cdot \psi_{\mathcal{U}}=0 \in \mathrm{H}_{\text {ét }}^{2}(U, Z)$ according to Lemma 7.4. This shows $n \cdot \psi_{G}^{d}=0$ generically.

Proof of Theorem 6.8. Consider a positive integer $n \geq 1$.

Suppose $n \cdot \psi_{G}^{d}=0$ in $\mathrm{H}_{\text {ét }}^{2}\left(\mathfrak{M}_{G}^{d, \text { rs }}, Z\right)$. Applying Lemma 7.4 to the map $n: Z \rightarrow Z$, we get a homomorphism $\sigma: \operatorname{Hom}\left(Z, \mathbb{G}_{\mathrm{m}}\right) \rightarrow \operatorname{Pic}\left(\mathcal{M}_{G}^{d, \mathrm{rs}}\right)$ with wt ${ }_{G}^{d} \circ \sigma=n$. Here the map $\mathrm{wt}_{G}^{d}$ vanishes on all torsion elements, according to Proposition 7.2 , i and Lemma 7.3 ,ii. It follows that $n$ annihilates the torsion $\operatorname{subgroup} \operatorname{Hom}\left(Z / Z^{0}, \mathbb{G}_{\mathrm{m}}\right)$ of $\operatorname{Hom}\left(Z, \mathbb{G}_{\mathrm{m}}\right)$.

Conversely, suppose that $n$ annihilates $\operatorname{Hom}\left(Z / Z^{0}, \mathbb{G}_{\mathrm{m}}\right)$, and $n \cdot \psi_{G}^{d}=0$ generically. Then there is an open subscheme $\emptyset \neq U \subseteq \mathfrak{M}_{G}^{d, r s}$ with

$$
n \cdot \psi_{\mathcal{U}}=0 \in \mathrm{H}_{\text {ét }}^{2}(U, Z), \quad \mathcal{U}:=\pi^{-1}(U) \subseteq \mathcal{M}_{G}^{d, \mathrm{rs}}
$$

We choose characters $\chi_{1}, \ldots, \chi_{r}: Z \rightarrow \mathbb{G}_{\mathrm{m}}$ whose restrictions to $Z^{0}$ form an isomorphism $Z^{0} \cong \mathbb{G}_{\mathrm{m}}^{r}$. Applying Lemma 7.4 to $n: Z \rightarrow Z$, and using Lemma 7.3., we can find line bundles

$$
\mathcal{L}_{1}, \ldots, \mathcal{L}_{r} \in \operatorname{Pic}\left(\mathcal{M}_{G}^{d, r s}\right)
$$

such that $\mathcal{L}_{i}$ has weight $n \chi_{i}$. We define $\sigma^{0}: \operatorname{Hom}\left(Z^{0}, \mathbb{G}_{\mathrm{m}}\right) \rightarrow \operatorname{Pic}\left(\mathcal{M}_{G}^{d, \mathrm{rs}}\right)$ by sending $\chi_{i}$ to $\mathcal{L}_{i}$. Then the homomorphism

$$
\sigma: \operatorname{Hom}\left(Z, \mathbb{G}_{\mathrm{m}}\right) \longrightarrow \operatorname{Pic}\left(\mathcal{M}_{G}^{d, \mathrm{rs}}\right), \quad \chi \mapsto \sigma^{0}\left(\left.\chi\right|_{Z^{0}}\right),
$$

satisfies the equation $\mathrm{wt}_{G}^{d} \circ \sigma=n$, because both sides of this equation vanish on $\operatorname{Hom}\left(Z / Z^{0}, \mathbb{G}_{\mathrm{m}}\right)$ and map $\chi_{i}$ to $n \chi_{i}$.

Due to Lemma 7.4, this implies $n \cdot \psi_{G}^{d}=0$ in $\mathrm{H}_{\text {ét }}^{2}\left(\mathfrak{M}_{G}^{d, r s}, Z\right)$. 


\section{REFERENCES}

[1] V. Balaji, I. Biswas, D.S. Nagaraj, and P.E. Newstead. Universal families on moduli spaces of principal bundles on curves. Int. Math. Res. Not., Article ID 80641, 2006.

[2] P. Bardsley and R.W. Richardson. Étale slices for algebraic transformation groups in characteristic p. Proc. Lond. Math. Soc., III. Ser., 51:295-317, 1985.

[3] I. Biswas and N. Hoffmann. The line bundles on moduli stacks of principal bundles on a curve. Documenta Math., 15:35-72, 2010.

[4] A. Borel. Properties and linear representations of Chevalley groups. In Seminar Alg. Groups and Related Finite Groups, Princeton 1968/69, Springer Lecture Notes in Mathematics 131, 1970.

[5] N. Bourbaki. Éléments de mathématique. Groupes et algèbres de Lie. Chapitres IV, V et VI. Paris: Hermann, 1968.

[6] N. Bourbaki. Éléments de mathématique. Groupes et algèbres de Lie. Chapitres VII et VIII. Paris: Hermann, 1975.

[7] R.W. Carter. Simple groups of Lie type. London etc.: John Wiley \& Sons, 1972.

[8] M. Demazure and P. Gabriel. Groupes algébriques. Paris: Masson et Cie, Éditeur; Amsterdam: North-Holland Publishing Company, 1970.

[9] M. Demazure and A. Grothendieck. SGA 3: Schémas en groupes. Exposés XIX à XXVI. Springer Lecture Notes in Mathematics 153, 1970.

[10] G. Faltings. Stable G-bundles and projective connections. J. Algebr. Geom., 2(3):507-568, 1993.

[11] J. Giraud. Cohomologie non abélienne. Grundlehren der mathematischen Wissenschaften, Band 179. Berlin-Heidelberg-New York: Springer-Verlag, 1971.

[12] T.L. Gómez, A. Langer, A.H.W. Schmitt, and I. Sols. Moduli spaces for principal bundles in arbitrary characteristic. Adv. Math., 219(4):1177-1245, 2008.

[13] T.L. Gómez, A. Langer, A.H.W. Schmitt, and I. Sols. Moduli spaces for principal bundles in large characteristic. In I. Biswas, R.S. Kulkarni, and S. Mitra, editors, Proceedings of 'Teichmüller Theory and Moduli Problems' (Allahabad, 2006), pages 281-371, 2010. Ramanujan Mathematical Society Lecture Notes Series, Vol. 10.

[14] J. Heinloth. Semistable reduction for G-bundles on curves. J. Algebr. Geom., 17:167-183, 2008.

[15] J. Heinloth. Addendum to "Semistable reduction for G-bundles on curves.". J. Algebr. Geom., 19:193-197, 2010.

[16] N. Hoffmann. Rationality and poincaré families for vector bundles with extra structure on a curve. Int. Math. Res. Not., 2007. article ID rnm010, 29 pages.

[17] N. Hoffmann. On Moduli Stacks of $G$-Bundles over a Curve. to appear in: A.H.W. Schmitt (Ed.), Proceedings of 'Affine Flag Manifolds and Principal Bundles' (Berlin 2008). Birkhäuser Trends in Mathematics, 2010. preprint available at http://userpage.fu-berlin.de/ nhoffman.

[18] Y. Holla. Parabolic reductions of principal bundles. preprint arXiv:math/0204219 available at http://www.arXiv.org.

[19] G. Laumon and L. Moret-Bailly. Champs algébriques. Ergebnisse der Mathematik und ihrer Grenzgebiete, Band 39. Berlin: Springer, 2000.

[20] D. Luna. Slices étalés. Bull. Soc. Math. Fr., Suppl., Mem., 33:81-105, 1973.

[21] P.E. Newstead. A nonexistence theorem for families of stable bundles. J. Lond. Math. Soc., II. Ser., 6:259-266, 1973.

[22] S. Ramanan. The moduli space of vector bundles over an algebraic curve. Math. Ann., 200:6984, 1973.

[23] M. Raynaud. Sections des fibrés vectoriels sur une courbe. Bull. Soc. Math. Fr., 110:103-125, 1982.

School of Mathematics, Tata Institute of Fundamental Research, Homi Bhabha Road, Mumbai 400005, India

E-mail address: indranil@math.tifr.res.in

Mathematisches Institut der Freien Universität, Arnimallee 3, 14195 Berlin, GerMANY

E-mail address: norbert.hoffmann@fu-berlin.de 